\title{
Diferenças na cinemática entre dois tipos de aterrissagens em atletas de voleibol masculinos
}

\author{
Difference in kinematical behavior between two landing tasks \\ in male volleyball athletes
}

\author{
Gustavo Leporace 1,2 \\ Jomilto Praxedes 1 \\ Rodrigo Fonseca 1 \\ Daniel Chagas 1,5 \\ João de Deus Brandão Júnior \\ Christian Rodrigues \\ Glauber Ribeiro Pereira \\ Luiz Alberto Batista 1,3,5
}

1. Universidade do Estado do Rio de Janeiro. Laboratório de Biomecânica e Comportamento Motor. Rio de Janeiro, RJ. Brasil.

2. Instituto Brasil de Tecnologias da Saúde. Rio de Janeiro, RJ. Brasil.

3. Universidade Estadual Paulista. Programa de Pós-Graduação em Engenharia Mecânica. São Paulo, SP. Brasil.

4. Universidade Castelo Branco. Programa de Pós Graduação em Motricidade Humana. Rio de Janeiro, RJ. Brasil.

5. Universidade do Estado do Rio de Janeiro. Programa de Pós-Graduação. Faculdade de Ciências Médicas. Rio de Janeiro, RJ. Brasil.

Recebido em 01/09/09 Revisado em 10/01/10 Aprovado em 18/05/10
Resumo - As lesões no ligamento cruzado anterior (LCA) são frequentes no âmbito esportivo. $\mathrm{O}$ estudo dos mecanismos de lesão demonstrou que a maioria das lesões é oriunda tanto de aterrissagens unilaterais quanto bilaterais. Apesar de estudos terem demonstrado diferenças no comportamento cinemático de membros inferiores entre homens e mulheres, não há evidências de como homens se comportam em diferentes condutas. O objetivo neste estudo foi comparar a cinemática angular e temporal dos membros inferiores, manifesta em aterrissagens de saltos com propulsão bilateral, realizadas sobre um e dois membros inferiores. Aterrissagens unilaterais e bilaterais no plano frontal e sagital de quinze atletas masculinos de voleibol foram registradas e processadas por meio de técnicas videogramétricas. Os resultados indicam que, na aterrissagem unilateral, os atletas apresentaram menor flexão do quadril e joelho, assim como maior valgismo do joelho, quando comparada com a aterrissagem bilateral. A diferença no tempo de aterrissagem entre as duas condutas não foi estatisticamente significante. Em conclusão, os resultados deste estudo apóiam a premissa de que a cinemática dos membros inferiores se altera em função da configuração da conduta motora realizada em homens. Estudos futuros são necessários para explorar o impacto das diferenças cinemáticas encontradas na tensão ligamentar e relacioná-las com os mecanismos de lesões no LCA em homens.

Palavras-chave: Cinemática; Aterrissagem; Risco de lesão.

Abstract - Anterior cruciate ligament (ACL) injuries are common in sports. Studies investigating injury mechanisms have demonstrated that most injuries arise from landing tasks. Despite the demonstration of differences between male and female kinematics, there are no studies showing how males behave during different landing tasks. The objective of this study was to compare the angular and temporal kinematics of the lower limbs between two different landing tasks. Double leg and single leg landings were recorded in the frontal and sagittal plane in 15 male volleyball athletes by videogrammetry. Reduced hip and knee flexion and increased knee valgus were observed in the single leg landing task compared to the double leg landing task. No significant difference in landing time was observed between the two tasks. In conclusion, the results support the premise that lower limb kinematics change according to the task performed. Further studies are necessary to explore the impact of these kinematic differences on knee loading and to relate them to ACL injury mechanisms in men.

Key words: Kinematics; Landing; Pubescent males; Injury risk. 


\section{INTRODUÇÃO}

A lesão musculoesquelética nos membros inferiores parece ser de ocorrência frequente no contexto da prática esportiva ${ }^{1}$. Como resultado de um estudo epidemiológico, Majewski et al. ${ }^{2}$ constataram que dentre as 19530 ocorrências investigadas, 7769 (39.8\%) estavam relacionadas à articulação do joelho. Dessas lesões, 3482 (44.8\%) eram traumas internos, dos quais, 1821 (52.3\%) implicaram danos ligamentares. No caso específico do Voleibol, que no referido estudo ocupou a $6^{a}$ posição em uma lista de 31 modalidades esportivas, organizadas por ordem crescente de índice de incidência de lesões do joelho, aproximadamente, $60 \%$ dos acometimentos às estruturas internas dessa articulação implicaram danos ao ligamento cruzado anterior (LCA).

Krosshaug et $\mathrm{al}^{3}$ e Santos et al. ${ }^{4}$ constataram que, para além do tipo de modalidade esportiva, a natureza da conduta motora realizada parece ser um fator importante no que concerne à incidência de lesões no LCA: Cerca de $60 \%$ das lesões no LCA decorrem de aterrissagens, sendo $26 \%$ em aterrissagens unilaterais e $34 \%$ em aterrissagens bilaterais ${ }^{3}$, resultado semelhante ao encontrado para lesões, em geral, em jogadores amadores de Voleibol ${ }^{4}$.

Ao que parece, aterrissagens unilaterais induzem a comportamentos motores diferentes dos comportamentos apresentados em aterrissagens bilaterais, o que possivelmente modifica o status de pré-disposição à instalação de lesões. Pappas et al..$^{5}$ demonstraram que os valores de diferentes variáveis biomecânicas, como as forças verticais de reação do solo (FVRS), mensuradas nos instantes de contato inicial e de pico do ângulo de flexão do joelho, são maiores em aterrissagens bilaterais, enquanto que o valgismo do joelho é maior durante a aterrissagem unilateral. Tillman et al. ${ }^{6}$ também encontraram maiores FVRS durante aterrissagens unilaterais em relação às bilaterais em mulheres.

Embora existam evidências de que as lesões no LCA, oriundas tanto de aterrissagens como de desacelerações bruscas, resultem de movimentos multiplanares, há suspeitas fundamentadas de que os fatores de risco e os mecanismos indutores de lesão são diferentes quando comparamos homens e mulheres ${ }^{3,7,8}$. Os mecanismos de lesão em mulheres mostram-se primariamente associados aos deslocamentos e cargas mecânicas manifestos nos planos sagital, frontal e transverso ${ }^{7}$, enquanto que, em homens, parecem estar primariamente relacionados com os movimentos e cargas de plano sagital ${ }^{9}$.

Se, por um lado, as informações provenientes desses estudos são importantes para o delineamento de estratégias de prevenção de lesões, por outro, sua generalização é limitada em decorrência da natureza da amostra e das variáveis biomecânicas investigadas. Apesar das evidências apontarem para diferenças de configuração entre os dois gestos esportivos ${ }^{5}$, os comportamentos de outras variáveis biomecânicas manifestas nos diferentes tipos de aterrissagem, também importantes na indução a quadros lesivos, como deslocamento angular, impulso e trabalho mecânico, não foram discutidos. Além disso, há o fato da escassez de estudos com a população masculina em estágios maturacionais diferentes.

O objetivo neste estudo foi comparar a cinemática angular e temporal dos membros inferiores, manifesta em aterrissagens de saltos com propulsão bilateral, realizadas sobre um e dois membros inferiores, por atletas de voleibol do sexo masculino púberes.

Nossa primeira hipótese era de que o quadril estaria mais flexionado no contato inicial e durante a angulação máxima, além do maior deslocamento angular na aterrissagem com dois membros inferiores. A segunda hipótese era de que o joelho estaria mais flexionado durante o contato inicial e no máximo, durante a aterrissagem bilateral, no entanto, o deslocamento angular seria semelhante entre as aterrissagens. A terceira hipótese era de que o valgismo do joelho máximo e no contato inicial seria maior na aterrissagem unilateral, sem diferenças para o deslocamento angular. A quarta hipótese era de que o tempo de aterrissagem, definido como o período de tempo entre o contato inicial do joelho e a angulação máxima, seria maior na aterrissagem bilateral.

\section{PROCEDIMENTOS METODOLÓGICOS}

Quinze atletas de voleibol (idade: $13 \pm 0.7$ anos, estatura: $170 \pm 12 \mathrm{~cm}$, massa corporal: $60 \pm 12 \mathrm{~kg}$ ), da categoria mirim, do sexo masculino, participaram deste estudo. Todos os responsáveis pelos participantes assinaram o Termo de Consentimento Livre e Esclarecido, autorizando a participação de seus descendentes. Esse estudo foi aprovado pelo Comitê de Ética da Universidade do Estado do Rio de Janeiro sob o parecer nº 053/2009.

Inicialmente, os atletas foram submetidos a um exame de maturação sexual de acordo com o protocolo de Tanner et al. ${ }^{10}$. Este teste avalia a maturação sexual através de observação e pontuação de critérios como pêlos púbicos e genitais em homens. 
Todos foram classificados como púberes de acordo com o protocolo proposto por Tanner et al. ${ }^{10}$.

Cada indivíduo realizou dois tipos de saltos verticais. Para cada um, os atletas realizaram a fase de propulsão com ambos os membros inferiores e aterrissaram com um membro inferior (aterrissagem unilateral) ou com os dois membros inferiores (aterrissagem bilateral). Foi realizada uma divisão de fases na qual o I4 é o instante de contato com o solo (CI) e o I5 é o instante de angulação máxima (Máx). O deslocamento angular foi calculado através da fórmula I5 - I4 (FIGURAS 1A e 1B).

Inicialmente, os atletas executaram as condutas motoras algumas vezes de forma a familiarizarem-se com as mesmas. Depois de familiarizados, cada atleta executou seis saltos solo a solo, sendo que em três utilizaram aterrissagens com um membro, o dominante, e nas outras três aterrissagens com os dois membros. As imagens de plano frontal e sagital das execuções foram capturadas e armazenadas. Os saltos foram realizados de forma aleatória para minimizar os possíveis efeitos da fadiga ou processo de aprendizagem. Foi utilizado um intervalo de um minuto entre as tentativas.

Duas câmeras Sony DCR HC 46, com uma frequência de amostragem de $30 \mathrm{~Hz}$, foram utilizadas no registro das imagens, sendo posicionadas

A

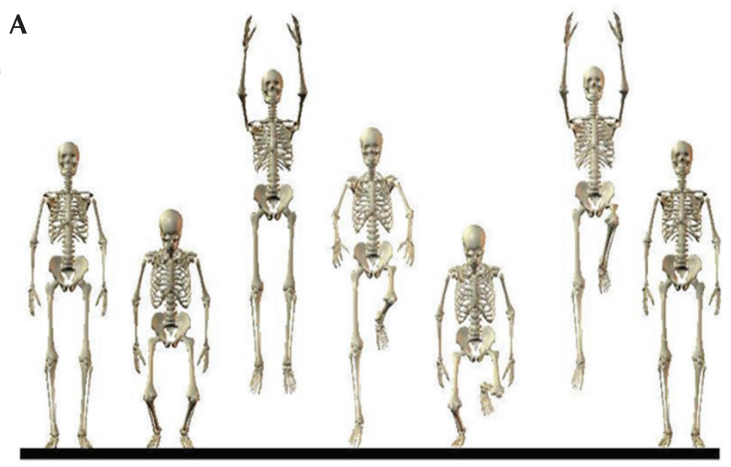

B

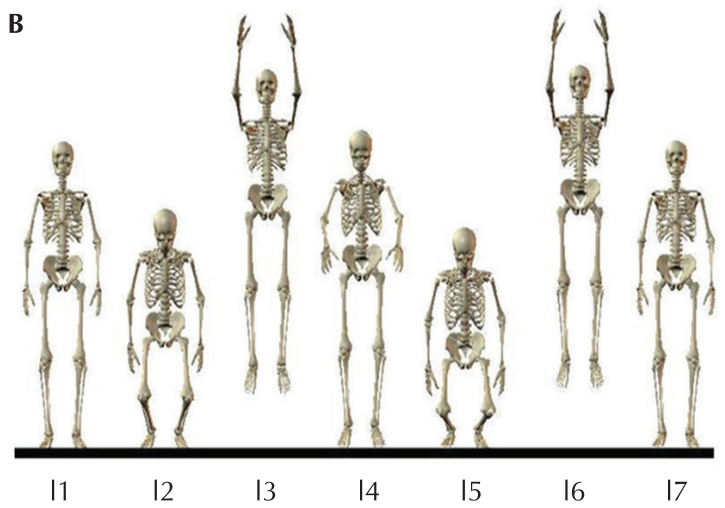

Figura 1: Ciclograma das aterrissagens. Nas duas condutas o salto inicial é realizado bilateralmente, seguido por uma aterrissagem unilateral (A) ou bilateral (B). a 2 metros de distância das executantes no plano sagital e frontal, com eixo ótico projetado sobre o centro da área de captura e perpendicular às suas orientações horizontal e vertical. Marcadores reflexivos esféricos de $20 \mathrm{~mm}$ foram fixados na crista ilíaca, no trocânter maior, no côndilo lateral do fêmur, no maléolo lateral, no calcâneo, na lateral do quinto metatarso, na região anterior da coxa (projeção da espinha ilíaca ântero-superior), no centro da patela e na tuberosidade da tíbia (FIGURA 2).

$\mathrm{O}$ modelo corporal formado pelos segmentos delimitados por esses marcadores foi utilizado na mensuração do comportamento angular e temporal das articulações do quadril e joelho no plano sagital e joelho no plano frontal.

Antes da realização dos testes, foi filmada a postura estática de cada indivíduo, visando calibrá-la com o sistema de coordenadas globais do ambiente de teste. $\mathrm{O}$ sistema de coordenadas locais de cada articulação foi alinhado com a postura estática, visando reduzir a variação intersujeitos em relação à posição fundamental. $\mathrm{O}$ ambiente de teste foi calibrado através da utilização de um cubo de medidas conhecidas.

Após a captura, as filmagens foram transferidas para um computador pessoal e os dados brutos das coordenadas dos marcadores transformados em coordenadas globais 2D através do método de Transformação Linear Direta ${ }^{11}$, processadas por meio do software Skillspector (versão 1.2.4) e os dados suavizados por meio de um filtro Quintic Spline com uma frequência de corte de $6 \mathrm{~Hz}$.

Para sincronizar as duas câmeras no domínio do tempo, foi utilizado um foot-switch no solado do tênis, na região do primeiro metatarso do executante, o qual, ao ser comutado, acionava dois sinalizadores luminosos posicionados no campo de captura das duas câmeras. Esses sinais também foram utilizados
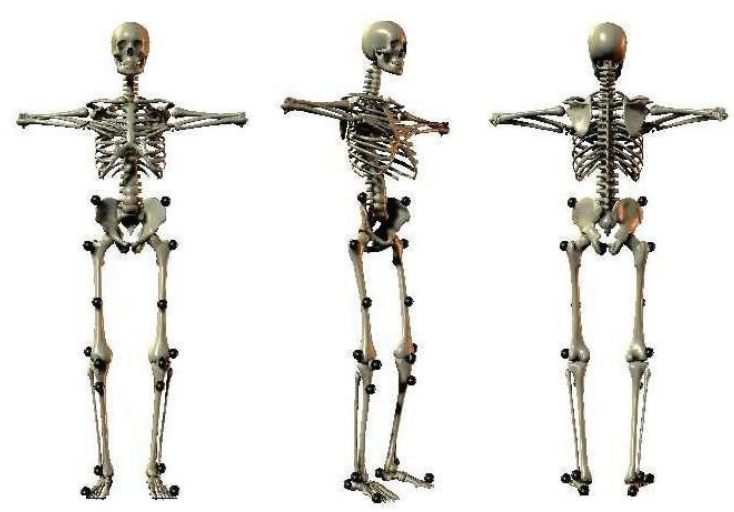

Figura 2: Posicionamento dos marcadores reflexivos esféricos nas regiões anatômicas descritas para mensuração da cinemática articular. 
para delimitar os instantes iniciais e finais da fase aérea, possibilitando identificar, para cada salto, o tempo dessa etapa, sendo esse valor utilizado para estimar o deslocamento vertical máximo nos saltos, quando da execução dos gestos esportivos, de acordo com o procedimento proposto por Leard et $\mathrm{al}^{12}$.

\section{Análise Estatística}

Na condição de variáveis dependentes, foram examinados os valores angulares medidos no instante de contato inicial, os valores angulares de pico, assim como os deslocamentos angulares das articulações do quadril e joelho no plano sagital e frontal e o tempo de aterrissagem. Esse último foi definido como sendo o período de tempo decorrido entre os instantes do contato inicial com o solo e de flexão máxima do joelho (I4 e I5, respectivamente, na Figura 1). O deslocamento angular foi calculado por meio da subtração do valor angular de pico e aquele obtido no instante do contato inicial. Consideramos como $0^{\circ}$ a posição de extensão total do joelho ${ }^{5,13,14}$ e determinamos o valgismo do joelho como o deslocamento medial do marcador posicionado na patela em relação aos marcadores da espinha ilíaca e tuberosidade da tíbia. Em alguns estudos, esse deslocamento angular no plano frontal é descrito como colapso medial da articulação do joelho ${ }^{13,15}$. Como procedimento de controle, foram comparadas as alturas obtidas nos saltos, na execução das duas aterrissagens, uma vez que a altura da queda pode influenciar no comportamento cinemático das articulações dos membros inferiores $^{16,17}$, sendo a mesma tratada por nós como variável interveniente. Para todas as variáveis descritas acima, foi utilizado o valor obtido através da média aritmética entre as três tentativas realizadas. A variável independente foi o tipo de aterrissagem, com um ou dois membros inferiores.

Para cada uma das variáveis dependentes, foi realizada a comparação entre as aterrissagens unilateral e bilateral, assim como a variável altura do salto. Para o exame da diferença, foi utilizado o Wilcoxon Ranked Test, tendo sido adotado um nível de significância de 5\% e processamento realizado por meio do software GraphPad Prism, versão 5.00 para Windows (GraphPad Software, San Diego California USA).

\section{RESULTADOS}

Instante de Contato com o Solo

Não foram encontradas diferenças significativas para a posição angular da articulação do quadril entre as duas aterrissagens nessa fase $(\mathrm{p}=0.8457)$. Já a articulação do joelho apresentou maior flexão $(\mathrm{p}=0.002)$ e menor valgismo $(\mathrm{p}=0.0049)$ na aterrissagem bilateral, em comparação com a aterrissagem unilateral.

Grau de Angulação Máximo:

As articulações do quadril e do joelho apresentaram maior flexão na aterrissagem bilateral do que na unilateral $(\mathrm{p}=0.002$ e $\mathrm{p}=0.002$, respectivamente). No plano frontal, a articulação do joelho apresentou menor valgismo na aterrissagem bilateral do que na unilateral $(\mathrm{p}=0.0029)$.

\section{Deslocamento Angular:}

$O$ deslocamento angular da articulação do quadril foi maior na aterrissagem bilateral do que na unilateral ( $\mathrm{p}=0.002$ ). Já para a articulação do joelho, tanto no plano frontal como no sagital, não houve diferenças estatisticamente significativas do deslocamento angular entre as aterrissagens $(\mathrm{p}=0.4131$ e $p=0.5566$, respectivamente).

\section{Tempo de aterrissagem:}

Não foram encontradas diferenças estatisticamente significativas $(p=0.202)$ entre as condutas motoras examinadas no que se refere ao tempo de aterrissagem.

\section{Altura dos Saltos}

A altura máxima alcançada foi maior na execução do salto com aterrissagem bilateral do que no de aterrissagem unilateral $(\mathrm{p}=0.0025)$.

Os dados numéricos (média e desvio padrão) estão descritos na tabela 1 .

Tabela 1: Valores angulares $\left({ }^{\circ}\right)$, temporais $(\mathrm{s})$ e espaciais $(\mathrm{m})$ nas duas aterrissagens.

\begin{tabular}{|c|c|c|c|c|}
\hline \multicolumn{2}{|c|}{ Variáveis Cinemáticas } & \multirow{2}{*}{$\frac{A U}{23,6 \pm 6,8}$} & \multirow{2}{*}{$\frac{A B}{23,6 \pm 8,1}$} & \multirow{2}{*}{$\begin{array}{c}\text { P valor } \\
0,8457\end{array}$} \\
\hline & $\mathrm{Cl}$ & & & \\
\hline $\begin{array}{l}\text { Flexão do Qua- } \\
\left.\text { dril ( }{ }^{\circ}\right)\end{array}$ & Máx** & $47,9 \pm 10,9$ & $73,3 \pm 13,8$ & 0,002 \\
\hline & $\boldsymbol{\Delta} \mathrm{s}^{* *}$ & $24,2 \pm 7,1$ & $49,6 \pm 19,8$ & 0,002 \\
\hline \multirow{3}{*}{$\begin{array}{l}\text { Flexão do } \\
\text { Joelho }\left(^{\circ}\right)\end{array}$} & $\mathrm{Cl}^{* *}$ & $9,9 \pm 5,0$ & $21,1 \pm 10,5$ & 0,002 \\
\hline & Máx** & $57,3 \pm 5,7$ & $70,9 \pm 7,0$ & 0,002 \\
\hline & $\boldsymbol{\Delta} \mathrm{s}$ & $47,3 \pm 4,2$ & $49,8 \pm 10,6$ & 0,4131 \\
\hline \multirow{3}{*}{$\begin{array}{l}\text { Valgismo do } \\
\text { Joelho }\left(^{\circ}\right)\end{array}$} & $\mathrm{Cl}^{* *}$ & $-2,7 \pm 7,4$ & $-8,5 \pm 7,9$ & 0,0049 \\
\hline & Máx** & $3,2 \pm 5,8$ & $-4,3 \pm 7,4$ & 0,0029 \\
\hline & $\boldsymbol{\Delta} \mathrm{s}$ & $6,0 \pm 3,9$ & $4,2 \pm 3,9$ & 0,5566 \\
\hline $\begin{array}{l}\text { Tempo de } \\
\text { Aterrissagem (s) }\end{array}$ & $\Delta \mathrm{t}$ & $0,30 \pm 0,07$ & $0,37 \pm 0,16$ & 0,202 \\
\hline $\begin{array}{l}\text { Altura do } \\
\text { Salto }(\mathrm{m})\end{array}$ & $\Delta \mathrm{h}^{* *}$ & $0,26 \pm 0,06$ & $0,30 \pm 0,07$ & 0,0025 \\
\hline
\end{tabular}

Resultados expressos pela média \pm desvio padrão. $\mathrm{AU}=$ Aterrissagem Unilateral, $\mathrm{AB}=$ Aterrissagem Bilateral, $\mathrm{Cl}=$ Contato inicial com o solo, representado pelo 14 da Figura 1, Máx=Angulação máxima, representado pelo I5 da Figura 1, $\boldsymbol{\Delta} \mathrm{s}=$ deslocamento angular entre $\mathrm{Cl}$ e Máx, $\boldsymbol{\Delta} \mathrm{t}=$ intervalo de tempo entre os instantes de $\mathrm{Cl}$ e Máx do joelho, $\boldsymbol{\Delta} \mathrm{h}=$ altura do salto, $* * p<0.01$. 


\section{DISCUSSÃO}

Nesse estudo demonstrou-se que o grupo examinado apresentou diferenças no comportamento cinemático entre aterrissagens com um e dois membros inferiores.

A veracidade de nossa primeira hipótese só pode ser parcialmente aceita, pois a perspectiva das diferenças estatísticas entre os dois tipos de aterrissagem, quando do exame do comportamento da articulação do quadril no instante do contato inicial, não foram identificadas. No entanto, foi observado, ainda para a articulação do quadril, maior pico de flexão e maior deslocamento angular na aterrissagem bilateral, com diferenças significativas, quando comparados com os valores médios obtidos no outro tipo de aterrissagem. É possível que esse comportamento seja explicado pelo fato de, na aterrissagem bilateral, os indivíduos contarem com a disponibilidade de uma maior área de base de sustentação, o que viabiliza um maior deslocamento horizontal do centro de massa corporal com menor risco de comprometer a condição de equilíbrio ${ }^{18}$. Há evidências de que, pelo fato do apoio em um só pé instituir uma condição de maior instabilidade, os indivíduos tendam a utilizar a estratégia de aumentar a rigidez do tronco e com isso tentar obter uma melhor estabilidade ${ }^{19}$. Aterrar com o tronco mais verticalizado implica atenuar a utilização do glúteo máximo na frenagem do deslocamento após o contato com o solo ${ }^{18}$, e a menor utilização dessa musculatura pode estar relacionada com alterações na cinemática do plano frontal, já que o glúteo máximo é um rotador externo do quadril ${ }^{12}$. Além disso, embora essa estratégia possibilite assegurar uma melhor condição de equilíbrio, Shimokochi et al. ${ }^{20}$ demonstraram que aterrar com o tronco ereto aumenta o torque extensor no joelho, o que, dentre outras coisas, tende a aumentar a tensão no LCA.

Nossa segunda hipótese mostrou-se verdadeira, pois no instante de contato com o solo, o joelho apresentou-se em maior flexão nas aterrissagens bilaterais, o mesmo ocorrendo para os valores de pico de flexão. Pappas et al. ${ }^{5}$ especularam que, durante aterrissagens unilaterais, essa menor flexão é um mecanismo compensatório com o objetivo de manter o quadríceps mais próximo de seu comprimento original, condição na qual ele é mais efetivo para desacelerar o corpo e absorver cargas mecânicas. No entanto, um aspecto a ser considerado é a diferença na altura dos saltos quando na realização de cada tipo de aterrissagem. Ao que parece, a articulação do joelho é a principal responsável na absorção e modulação das forças de reação do solo ${ }^{21}$. Apesar de a fase de propulsão do salto, para os dois tipos de aterrissagem, ter sido realizada com os dois membros inferiores (Figura 1A e 1B), nas situações em que houve aterrissagens bilaterais, os indivíduos alcançaram maior deslocamento vertical, o que, possivelmente, gerou maior energia mecânica nessa condição, em comparação com as condições de aterrissagem unilateral. Não encontramos na literatura dados que justificassem esses achados, no entanto, supomos que o menor deslocamento vertical seja estratégico no que tange à atenuação das FVRS e dessa maneira facilitam a obtenção da estabilidade na aterrissagem unilateral, uma vez que as condições de aterrissagens eram conhecidas antes do início da fase de propulsão.

Em tese, essa maior quantidade de energia mecânica é contrabalanceada pelo fato da modulação das cargas ser realizada com os dois membros inferiores, ao contrário da condição de aterrissagem unilateral, na qual, apesar de menor energia mecânica envolvida, o trabalho mecânico para modular as cargas deve ser realizado por apenas um membro inferior, o que pode justificar o deslocamento angular semelhante nas duas condições. Estudos futuros são necessários para comprovar essa pressuposição.

Apesar de no gesto esportivo unilateral a energia gerada no salto ser menor devido à menor altura alcançada, a absorção dessa energia foi realizada com metade da estrutura corporal disponível para o ato, para além do fato de que a articulação do joelho apresentou-se, nos instantes iniciais e finais da aterrissagem, em maior extensão, contribuindo com o potencial deletério dessa etapa do salto. Pandy $\&$ Shelbourne ${ }^{22}$ e Shelbourne \& Pandy ${ }^{23}$ demonstraram, por meio de modelos computacionais, que com o joelho próximo à extensão o LCA está mais susceptível a tensões potencialmente lesivas e, de fato, mais recentemente, estudos observacionais possibilitaram a constatação de que no instante da lesão do LCA, o joelho apresentava maior valor de extensão $0^{3,24,25}$.

No plano frontal, os resultados estatisticamente significativos demonstraram a veracidade de nossa terceira hipótese. Um maior valgismo do joelho na aterrissagem unilateral foi perspectivado com base no fato de que a disposição anatômica implicada nesse ato propicia um aumento dos graus de liberdade da pelve, dificultando a estabilização da mesma. Além disto, existem achados na literatura demonstrando estes resultados ${ }^{4}$. Apesar de não haver evidências de que o valgismo faça parte do mecanismo de lesão em homens ${ }^{9}$, Markolf et al. ${ }^{26}$ demonstraram que aumentos no valgismo do joe- 
lho, tal como o que foi observado na aterrissagem unilateral, aumentam as magnitudes das forças tensivas no LCA, consistindo em um maior potencial de risco de lesões nesse componente anatômico. Em estudo recente, Herrington e Munro ${ }^{15}$ identificaram valores normativos de valgismo, quando avaliados em 2D, como é o caso do presente estudo, e demonstraram que homens apresentam até $8^{\circ}$ e $9^{\circ}$ de valgismo, quando na realização de aterrissagens bilaterais e unilaterais, respectivamente. Concluíram que valores acima desses poderiam impor riscos às estruturas ligamentares do joelho. Dessa forma, acreditamos que, mesmo com diferenças entre as aterrissagens, os valores de valgismo apresentados pelos atletas desse estudo não sugerem riscos aumentados para lesões no LCA.

No tocante ao tempo de aterrissagem, como havíamos previsto, não encontramos diferenças estatisticamente significativas entre as duas condutas motoras. Apesar de não termos mensurado as forças verticais de reação do solo (FVRS), existem evidências de que as aterrissagens unilaterais produzem maiores FVRS do que aterrissagens bilaterais ${ }^{6}$. Partindo dessa premissa, maiores cargas mecânicas deverão ser absorvidas no mesmo período de tempo que na aterrissagem bilateral. Como o quadríceps é o principal responsável pela desaceleração da articulação do joelho, é razoável que haja uma maior ativação desse músculo na aterrissagem unilateral ${ }^{6}$ e essa maior atividade muscular do quadríceps para frenar o movimento em menor tempo pode implicar um maior aumento na tensão do LCA nesse tipo de aterrissagem, em comparação com a bilateral. No entanto, não pudemos comprovar essa tese, uma vez que não mensuramos as FVRS e tendo em vista que a diferença na altura dos saltos, entre as duas condições examinadas, pode também alterar o comportamento dessas forças ${ }^{16,17}$.

Percebemos como limitações do presente estudo, a natureza da seleção da amostra, o baixo $\mathrm{N}$ amostral e a realização dos testes em ambientes laboratoriais. Em relação a essa última limitação, há evidências de que condutas motoras realizadas em laboratórios tendem a gerar quadros biomecânicos diferentes dos manifestos em situações reais de jogo ${ }^{27}$. Diante disso, julgamos ser necessário a realização de estudos futuros com amostras de maior $\mathrm{N}$, selecionadas aleatoriamente e com desenhos de teste que se aproximem ao máximo do contexto ambiental no qual os gestos esportivos são, efetivamente, utilizados, para que possamos inferir, com maior precisão, o impacto das diferenças cinemáticas na tensão ligamentar e relacioná-las com os mecanismos de lesões no LCA em homens esportistas.
Outra limitação foi a ausência no controle da altura do salto, variável que, comprovadamente, altera o comportamento cinemático durante gestos motores ${ }^{16,17}$. No entanto, o objetivo desse estudo foi discutir dois gestos comumente utilizados durante a prática esportiva do voleibol e por isso não adotamos outros tipos de salto, como o drop jump, no qual tal variável teria menor poder de interveniência.

\section{CONCLUSÃO}

No âmbito de validade da investigação aqui relatada, identificamos importantes diferenças cinemáticas no comportamento dos membros inferiores, quando comparamos dois tipos distintos de gestos esportivos, usualmente utilizados na chegada ao solo após a fase aérea de um salto vertical. Destarte, concluímos que, na aterrissagem unilateral, os atletas apresentaram os quadris e joelhos mais estendidos e maior valgismo do joelho, situações descritas na literatura como potencialmente lesivas para o LCA. Tais achados ratificam a proposição de que, em saltos verticais de jovens púberes do sexo masculino, as aterrissagens unilaterais tendem a induzir a conformação de posturas que podem levar a aumentos na tensão do LCA, apesar do baixo risco de se lesionarem, uma vez que os valores obtidos encontram-se dentro dos limites de normalidade.

\section{Agradecimentos}

Os autores agradecem à equipe do Laboratório de Biomecânica e Comportamento Motor, composta por Edgar Ingrácio, Douglas Aquino, Priscilla Christina e Paulo Pereira pelo auxílio na coleta de dados.

\section{REFERÊNCIAS BIBLIOGRÁFICAS}

1. Orchard J, Seward H. Epidemiology of injuries in the Australian Football League, seasons 1997-2000. Br J Sports Med 2002;36(1):39-45.

2. Majewski M, Susanne H, Klaus S. Epidemiology of athletic knee injuries: A 10-year study. The Knee 2006;13(3):184-8.

3. Krosshaug T, Nakamae A, Boden BP, Engebretsen L, Smith G, Slauterbeck JR, et al. Mechanisms of anterior cruciate ligament injury in basketball: video analysis of 39 cases. Am J Sports Med 2007;35(3):359-67.

4. Santos SG, Piucco T, Reis DC. Fatores que interferem nas lesões de atletas amadores de voleibol. Rev Bras Cineantropom Desempenho Hum 2007;9(2):189-95.

5. Pappas E, Hagins M, Sheikhzadeh A, Nordin M, Rose D. Biomechanical differences between unilateral and bilateral landings from a jump: gender differences. Clin J Sport Med 2007;17(4):263-8. 
6. Tillman MD, Criss RM, Brunt D, Hass CJ. Landing Constraints Influence Ground Reaction Forces and Lower Extremity EMG in Female Volleyball Players. J Appl Biomech 2004;20(1):38-50.

7. Hewett TE, Zazulak BT, Myer GD, Ford KR. A review of electromyographic activation levels, timing differences and increased anterior cruciate ligament injury incidence in female athletes. Br J Sports Med. 2005;39(6):347-50.

8. Zazulak BT, Hewett TE, Reeves NP, Goldberg B, Cholewicki J. Deficits in neuromuscular control of the trunk predict knee injury risk: a prospective biomechanical-epidemiologic study. Am J Sports Med. 2007;35(7):1123-30.

9. Quatman CE, Hewett TE. The ACL Injury Controversy: Is "Valgus Collapse" a Sex-Specific Mechanism? Br J Sports Med 2009;43(5):328-45.

10. Tanner JM. Growth at adolescence. Oxford: Blackwell, 1962.

11. Challis JH, Kerwin DG. Accuracy assessment and control point configuration whin using DLT for photogrammetry. J Biomech 1992; 25(9):1053-8.

12. Leard JS, Cirillo MA, Katsnelson E, Kimiatek DA, Miller TW, Trebincevic K, Garbalosa JC. Validity of two alternative systems for measuring vertical jump height. J Strength Cond Res. 2007;21(4):1296-9.

13. McLean SG, Walker K, Ford KR, Myer GD, Hewett TE, van den Bogert AJ. Evaluation of a two dimensional analysis method as a screening and evaluation tool for anterior cruciate ligament injury. Br J Sports Med 2005;39(6):355-362.

14. Neumann DA. Kinesiology of the musculoskeletal system. Foundations for physical rehabilitation. Saint Louis: Mosby, 2002.

15. Herrington L, Munro A. Drop jump landing knee valgus angle; normative data in a physically active population. Phys Ther Sport. 2010;11(2):56-9.

16. Yeow CH, Lee PV, Goh JC. Sagittal knee joint kinematics and energetics in response to different landing heights and techniques. Knee. 2009;17(2):127-31.

17. Yeow CH, Lee PV, Goh JC. Effect of landing height on frontal plane kinematics, kinetics and energy dissipation at lower extremity joints. J Biomech. 2009;42(12):1967-73. 2009;

18. Pollard CD, Sigward SM, Powers CM. Gender differences in hip joint kinematics and kinetics during side-step cutting maneuver. Clin J Sport Med 2007;17(1):38-42.
19. Zazulak BT, Ponce PL, Straub SJ, Medvecky MJ, Avedisian L, Hewett TE. Gender comparison of hip muscle activity during single-leg landing. J Orthop Sports Phys Ther 2005,35(5):292-9.

20. Shimokochi Y, Yong Lee S, Shultz SJ, Schmitz RJ. The relationships among sagittal-plane lower extremity moments: implications for landing strategy in anterior cruciate ligament injury prevention. J Athl Train 2009;44(1):33-8.

21. Elmer SJ, Madigan ML, LaStayo PC, Martin JC. Joint-specific power absorption during eccentric cycling. Clin Biomech 2010;25(2):154-158.

22. Pandy MG, Shelbourne KB. Dependence of cruciate-loading on muscle forces and external load. J Biomech 1997;30(10):1015-1024.

23. Shelbourne KB, Pandy MG. A musculoskeletal model of the knee for evaluating ligament forces during isometric contractions. J Biomech 1997;30(2):163-76.

24. Olsen OE, Myklebust G, Engebretsen L, Bahr R. Injury mechanisms for anterior cruciate ligament injuries in team handball: a systematic video analysis. Am J Sports Med 2004;32(4):1002-12.

25. Shimokochi Y, Shultz SJ. Mechanisms of noncontact anterior cruciate ligament injury. J Athl Train 2008;43(4):396-408.

26. Markolf KL, Burchfield DM, Shapiro MM, Shepard MF, Finerman GA, Slauterbeck JL. Combined knee loading states that generate high anterior cruciate ligament forces. J Orthop Res 1995;13(6):930-5.

27. Lian O, Engebretsen L, Ovrebo RV, Bahr, R. Characteristics of the leg extensors in male volleyball players with jumper's knee. Am J Sports Med 1996;24(3):380-5.

\section{Endereço para correspondência \\ Laboratório de Biomecânica \\ e Comportamento Motor \\ Universidade do Estado do Rio de Janeiro \\ Rua São Francisco Xavier, 524, Maracanã $8^{\circ}$ andar, sala 8122 .}

CEP: 20550-900 - Rio de Janeiro - Brasil

E-mail: guleporace@yahoo.com.br 